\title{
AFM Characterization of Biomolecules in Physiological Environment by an Advanced Nanofabricated Probe
}

\author{
M. MORETTI,${ }^{1}$ C. CANALE,${ }^{1}$ M. FRANCARDI ${ }^{2}$ S. DANTE,${ }^{1}$ F. DE ANGELIS,${ }^{1}$ AND E. DI FABRIZIO ${ }^{1,2}$ \\ ${ }^{1}$ Nanostructures Department, Istituto Italiano di Tecnologia, Via Morego 30, 16163 Genova, Italy \\ ${ }^{2}$ BIONEM Lab, Department of Experimental and Clinical Medicine, University of Magna Graecia, Campus S. Venuta, \\ Germaneto, viale Europa, 88100 Catanzaro, Italy
}

\author{
KEY WORDS AFM; nanofabrication; Raman spectroscopy; high-resolution topography; \\ biological molecules
}

\begin{abstract}
Many relevant questions in biology and medicine require both topography and chemical information with high spatial resolution. Several biological events that occur at the nanometer scale level need to be investigated in physiological conditions. In this regard Atomic Force Microscopy (AFM) is one of the most powerful tools for label-free nanoscale characterization of biological samples in liquid environment. Recently, the coupling of Raman spectroscopy to scanning probe microscopies has opened new perspectives on this subject; however, the coupling of quality AFM spectroscopy with Raman spectroscopy in the same probe is not trivial. In this work we report about the AFM capabilities of an advanced high-resolution probe that has been previously nanofabricated by our group for coupling with Raman spectroscopy applications. We investigate its use for liquid AFM measurements on biological model samples like lipid bilayers, amyloid fibrils, and titin proteins. We demonstrate topography resolution down to nanometer level, force measurement and stable imaging capability. We also discuss about its potential as nanoscale chemical probe in liquid phase. Microsc. Res. Tech. 00:000-000, 2012. @ 2012 Wiley Periodicals, Inc.
\end{abstract}

\section{BACKGROUND}

A plethora of biological events involved in biological systems such as enzymatic activity, adhesion, cell-cell signaling, membrane trafficking, and so on, occur in highly localized regions and can involve very few molecules. The effort to understand and intervene on these mechanisms has widely involved the field of nanofabrication at many levels (Liberale et al., 2007; Pérennès et al., 2006; Tormen et al., 2004; Yu et al., 2005), and among the other, drug delivery, transmembrane transport, diagnosis of single/few molecules (Das et al., 2009; De Angelis et al., 2010a,b, 2011a,b; Moretti et al., 2008). The need to obtain nanometer scale analytical data in physiological conditions is crucial to answer questions on biologically active complexes. In this regard atomic force microscopy (AFM) is one of the most powerful tools for nanoscale characterization of biological samples in water solutions. AFM provides the opportunity to study the sample in a noninvasive manner, limiting the need of fixation or dehydration, with the advantage to follow sample evolution, monitoring the structural changes in real-time, with the limitation imposed by the typical timescale of AFM experiments. The role of the probe in SPM is of primary importance to obtain high quality images of biological samples. The capability to discriminate two small objects (with a lateral size of few nanometers) depends on the tip radius of curvature, which permits the in plane resolution, while a high aspect-ratio probe provides a minimization of the tip broadening and deformation on thick and sharp samples. However, tip artifacts ever affect sample topography acquired by using AFM (Canale et al., 2011). In particular, the degree of broadening in the case of objects that are smaller with respect to the AFM tip depends on the radius of curva- ture of the tip apex, while taller objects, such as cells, can interact also with the tip side (Velegol et al., 2003). To minimize these effects that reduce the real resolution of the AFM, several fine tips have been fabricated using specialty techniques: FIB milling for sharpening of conventional silicon tips, electron beam growth deposition, gluing or carbon nanotubes deposition on top of a standard tip, assembly of cantilevers on cantilevers [(Yacoot and Koenders, 2008) and references therein]. Additionally, probes get easily contaminated both by hydrocarbons in air and by debris from biological samples: the contamination layer usually determines loss of image and force spectroscopy quality (DeRose, 1998; Dufrêne et al., 2011). The AFM capabilities are not limited to the topographical analysis: this instrument is a highly sensitive force sensor and it has been used in the last years to probe the strength of intermolecular interactions or the mechanical properties of biological samples, working at the single molecule level in a physiological environment (Kienberger et al., 2005; Oberhauser et al., 1998; Puchner and Gaub, 2009; Wong, 1999). However, although the use of functionalized tips with specific molecular groups allowed for molecular recognition in heterogeneous samples (Allen et al., 1997; Hinterdorfer, 1996; Stroh et al., 2004), also including cells (Chtcheglova et al., 2007), it could not

\footnotetext{
Author contributions: Manola Moretti and Claudio Canale equally contributed to the work presented in this article.

*Correspondence to: M. Moretti, Istituto Italiano di Tecnologia, Via Morego 30 16163 Genova, Italy. E-mail: manola.moretti@iit.it

Received 19 July 2012; accepted in revised form 9 August 2012

Contract grant sponsor: European Projects SMD FP7; Contract grant number: CP-FP 229375-2; Contract grant sponsor: Nanoantenna FP7; Contract grant number: 241818; Contract grant sponsor: FOCUS FP7; Contract grant number: 270483

DOI 10.1002/jemt.22122

Published online in Wiley Online Library (wileyonlinelibrary.com).
} 
overcome the lack of a direct chemical analysis on the sample and this is one of the great limitations of bare AFM technique. To this end, promising applications for the generation of a controlled surface plasmon polariton field at the AFM tip apex (Bek et al., 2010; De Angelis et al., 2008, 2010a, 2011a,b) have recently emerged. The enhanced electromagnetic field, highly confined in the proximity of the probe apex (in volumes in the order of thousands of $\mathrm{nm}^{3}$ ) allows for the detection of a Raman signal arising from the small number of molecules that are present in the nanometric excitation volume, opening the way for a real single molecule Raman spectroscopy. New kinds of nanoantennas with increased enhancing efficiency have been recently developed (Descrovi et al., 2009; Fleischer et al., 2010; Kharintsev et al., 2011; Stadler et al., 2010; Weber-Bargioni et al., 2011), but not all of these devices are suitable for high resolution AFM application.

This work will demonstrate the remarkable AFM capabilities of a recently nanofabricated probe on biological samples in liquid environment. The high-resolution performance and stability of the probe will pave the way for a coupled SPM/Raman topography in the nanometer range resolution. In the following chapters, we briefly report on the nanofabrication procedure of a high aspect-ratio $\mathrm{Pt} / \mathrm{C}$ nanocone with small tip radius of curvature that has been demonstrated to produce a high intensity plasmon polariton field (De Angelis et al., 2010a). We demonstrate in detail the advancement of the tip characterization under the scanning probe microscopy (SPM) point of view, which is a crucial step toward the integration of the microscopy and spectroscopy techniques. In particular we prove the nanoscale topographical resolution and the ability to work on delicate biological samples, operating in liquid environment and using different AFM acquisition modes (contact mode, tapping mode) with high quality and reproducible results. To demonstrate that a lateral resolution improvement on thick and sharp structures is achievable by using high aspect-ratio nanocone probes, we acquired images on a standard silicon calibration grating. Afterward, we investigated the morphology and the mechanical properties of model membranes supported on mica to check the probe capability to work on biological samples with undamaging interactions. Finally we examined the structural features of insulin amyloid fibrils with the aim to demonstrate an improved topographical resolution on soft samples in liquid environment. To check the eventual effects of the nanocone fabrication procedure on the cantilever properties, we tested our probes performing a typical single molecule experiment: the unfolding of the model derivative of the polyprotein Titin Ig27.

\section{MATERIALS AND METHODS AFM Probe}

An AFM tip suitable for nanometer resolution topography and Raman spectroscopy was developed according to the protocol reported in a previous paper of De Angelis et al. (2010a). The nanocone is fabricated on the apex of a triangular tipless AFM cantilever (Silicon Nitride probes from NanoandMore, $30-75 \mathrm{kHz}$ air resonance frequency and 0.32 and $0.06 \mathrm{~N} \mathrm{~m}^{-1}$ nominal spring constant) that was selected to measure soft samples in liquid (Fig. 1a). As reported, the plasmonic antennas result from a combination of nanolithography techniques: focused ion beam (FIB) milling and chemical vapor deposition induced by a focused electron beam, usually called EBID (electron beam induced deposition). Because here we are interested to test nanocones as AFM probes, we focused the attention on the fabrication of the tip only, without the photonic crystal only necessary for Raman spectroscopy applications (De Angelis et al., 2010a). A gas precursor containing Platinum $\left(\left(\mathrm{CH}_{3}\right)_{3} \mathrm{Pt}\left(\mathrm{C}_{\mathrm{p}} \mathrm{CH}_{3}\right), \mathrm{C}_{\mathrm{p}}\right.$ : cyclo-pentadiene) is injected into the SEM chamber. Electron beam with energy of $30 \mathrm{kV}$ and current of $100 \mathrm{pA}$ is used to grow the nanocone in the desired position. The deposited material consists in a mixture of platinum nanocrystals, embedded in a matrix of amorphous carbon with a relative percentage in weight of about 30 and $70 \%$, respectively (De Angelis et al., 2011b). Typical nanocone has a height of $2.5 \mu \mathrm{m}$ and radius of curvature of the tip apex of $5 \mathrm{~nm}$ (Fig. $1 \mathrm{~b}$ and related inset zoomed picture). Later the platinum/carbon nanocone can be covered with a gold layer (or other metal) when necessary. The deposition of few nm of gold by means of conventional thermal evaporation does not affect significantly the shape of the nanocone.

The probe is used as such for topography imaging, or it is evaporated with a $10 \mathrm{~nm}$ gold layer for force-distance measurements.

\section{Lipid Bilayers}

The supported lipid bilayers (SLB) were formed on mica substrate using the lipid vesicles fusion method (Mingeot-Leclercq et al., 2008). The binary mixture 1palmitoyl-2-oleoyl-sn-glycero-3-phosphocholine/1,2-

dipalmitoyl-sn-glycero-3-phosphocoline (POPC/DPPC) (1:1 molar ratio) was dissolved at $1 \mathrm{mg} \mathrm{mL}^{-1}$ in chloroform. Aliquots of $1 \mathrm{mg}$ in glass tubes were $\mathrm{N}_{2}$ dried, and then the pellet was resuspended in $1 \mathrm{~mL}$ PBS $1 \times$ buffer at $60^{\circ} \mathrm{C}$ in a water bath. The lipid solution was then filter-passed 11 times (through $50 \mathrm{~nm}$ polycarbonate filter membranes) using a commercial extruder (Avanti Polar Lipids, Alabaster, AL). A drop of the solution containing the newly formed $50 \mathrm{~nm}$ lipid vesicles was deposited on freshly cleaved mica and incubated for $1 \mathrm{~h}$ in a humid chamber to allow vesicle fusion and lipid bilayer formation. The substrate was gently rinsed with MilliQ water right before AFM measurement.

\section{Amyloid Fibrils}

Mature amyloid fibrils were obtained from bovine insulin ( $5 \mathrm{mg} \mathrm{mL}^{-1}$ ) (Sigma-Aldrich). Protein solution was incubated in $\mathrm{HCl}$ water solution (MilliQ filtered water) at $\mathrm{pH} 2.00$ for 3 weeks at $60^{\circ} \mathrm{C}$. A drop of $20 \mu \mathrm{L}$ of a 1:10 diluted solution was pipetted onto freshly cleaved mica substrate. After $15 \mathrm{~s}$ the sample was rinsed with $5 \mathrm{~mL}$ of MilliQ water and placed under the microscope for measurement.

\section{Titin}

The I27O synthetic polyprotein (Athena Enzyme Systems, MD) was used as reference protein for force extension curves. The protein solution was brought to a concentration of $60 \mu \mathrm{g} \mathrm{mL} \mathrm{mL}^{-1}$ in PBS $1 \times$ buffer and $20 \mu \mathrm{L}$ were deposited on freshly cleaved mica. The solution was at rest for $20 \mathrm{~min}$ in a humid chamber and then gently rinsed with $\mathrm{PBS} 1 \times$, ready for measurement. 


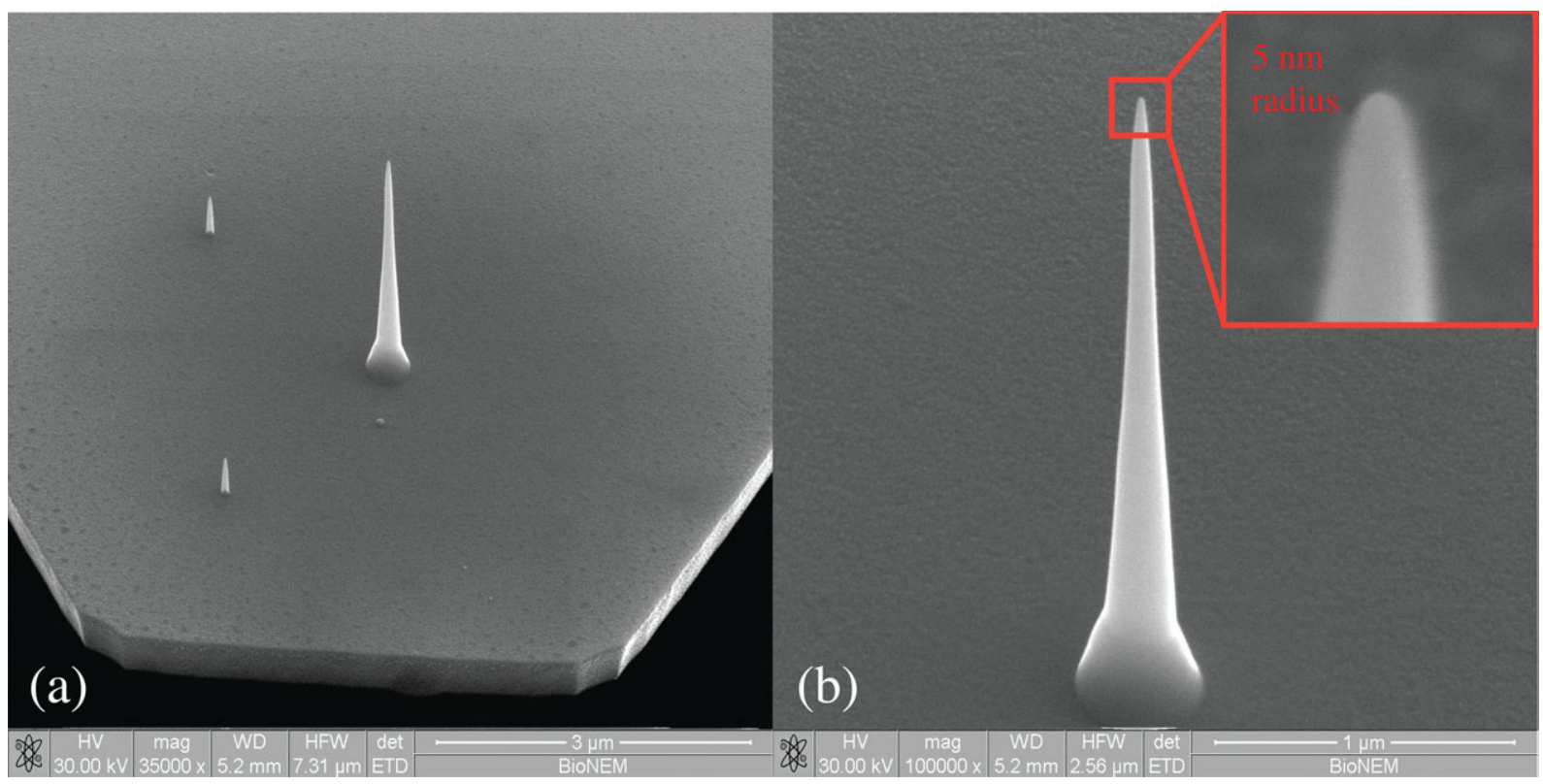

Fig. 1. Scanning electron micrograph of typical nanocone tip grown on triangular cantilever (a) and related zoomed picture (b) with indicated radius of curvature of the tip apex (red box). [Color figure can be viewed in the online issue, which is available at wileyonlinelibrary.com.]

\section{AFM Imaging and Force Spectroscopy}

Calibration grating (3 $\mu \mathrm{m}$ step, $500 \mathrm{~nm}$ height, NTMDT, Moscow, Russia) and insulin samples images were recorded with the Multimode AFM head (Bruker) equipped with a Nanoscope $\mathrm{V}$ controller operating in tapping mode in deionized water. Imaging and force measurements on lipid bilayers and titin were performed with the Nanowizard II (JPK Instruments) mounted on Axio Observer D1 (Carl Zeiss, Germany) inverted optical microscope. In force spectroscopy on lipid bilayers the force/distance curves were acquired applying a maximum force in the range of $9 \mathrm{nN}$, with a curve length of $200 \mathrm{~nm}$ and a constant tip velocity of $1 \mu \mathrm{m} \mathrm{s}^{-1}$. Unfolding of I270 model protein was achieved setting the tip pulling velocity at $0.5 \mu \mathrm{m} \mathrm{s}^{-1}$ after contact of the tip to the surface with an applied force of $0.3 \mathrm{nN}$. Triangular silicon nitride cantilevers (Bruker NP silicon nitride probes for contact and fluid tapping mode) with nominal spring constant of $0.32 \mathrm{~N} \mathrm{~m}^{-1}$ were used for AFM imaging. Softer cantilevers, $0.06 \mathrm{~N} \mathrm{~m}^{-1}$, were used in force measurements. The actual spring constant of each cantilever was determined in situ, using the thermal noise method (Hutter and Bechhoefer, 1993).

\section{Data Analysis}

AFM images were subjected to a first order flattening when needed, and to spikes and streaks removal with a resolution of two pixels, using the software provided by the AFM in use. Gwyddion freeware software was used for images final editing in coherent graphics. A Gaussian fit on peaks and baseline of the fibrils longitudinal profile was made using OriginPro8. Step height of lipid bilayers was calculated using a staircase Gaussian fitting provided by Gwyddion. A polymer chain model (worm-like chain) was fitted to the force data to determine the length and the elastic properties of I27O model protein being stretched.

\section{RESULTS AND DISCUSSION}

The main goal of this study was the performance evaluation under the SPM microscopy point of view, of an AFM probe fabricated for future combined Raman spectroscopy applications. To study biological samples in their natural environment, we chose to nanofabricate the nanocone on soft silicon nitride triangular cantilevers. All the experiments mentioned in this article will demonstrate the stability of the combination chosen. First, the quality of a high aspect/ratio probe was evaluated on a standard calibration grating. A brief description of the biological samples characteristic will precede each measurement description.

\section{AFM Probe}

As a high aspect-ratio probe, our tip can be successfully used to perform image topography on sharp and thick structures without loosing lateral resolution. On the contrary commercial state of the art pyramidal silicon tips with etched side angle of $35.6^{\circ}$ from the normal, will contain in the image topography the convolution with the pyramidal geometry and will be limited in resolving high aspect ratio features. We performed height topography on a calibration grating having a 3 $\mu \mathrm{m}$ step between features and nominal height of 500 $\mathrm{nm}$ to verify this point. The measurement was performed in water immersion to mimic biological environment. Height topography of the grating acquired with pyramidal tips, showed both backward and forward profile with edges angle close to $35^{\circ}$. Height topography was acquired at the same rate using nanocone tips: both forward and backward signals showed a scan profile with edges angle close to $90^{\circ}$. Moreover, the image de-convolution, gave a half-cone angle estimation of $8^{\circ}$. This is reasonable as long as the $2.5 \mu \mathrm{m}$ height tip develops starting from a $300 \mathrm{~nm}$ circular base to the ending radius of curvature of mean nominal value of 
(a)
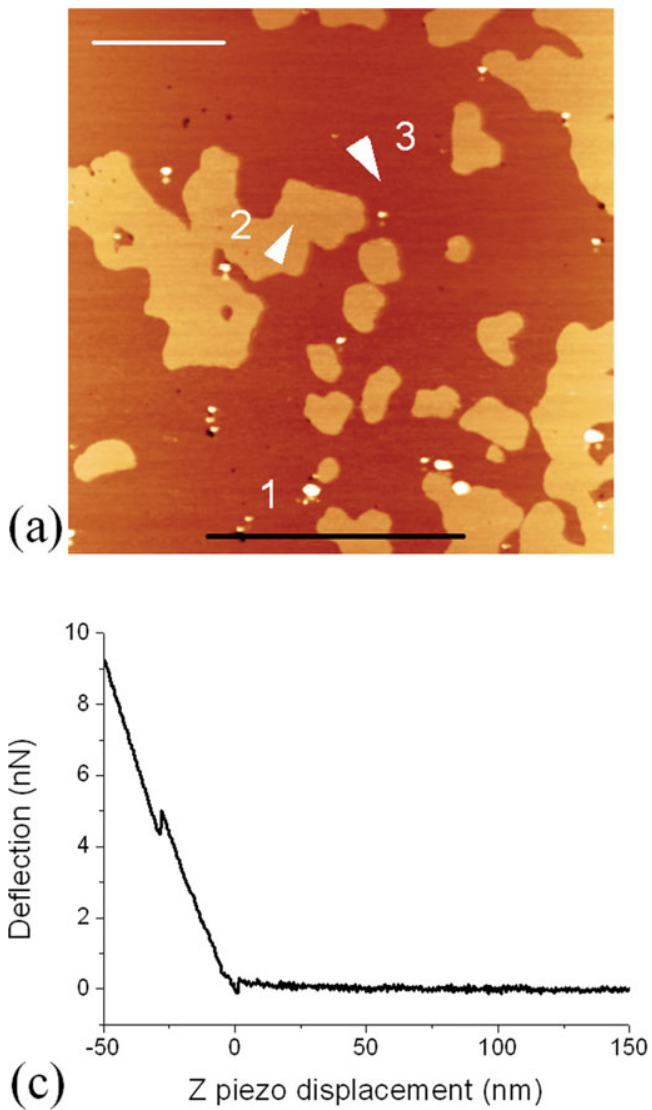

Fig. 2. From left to right: (a) AFM topography in liquid of POPC/ DPPC lipid bilayer formed on mica substrate, (b) profile [corresponding to profile 1 in (a)] of the sample evidencing the step height among substrate, lipid in the liquid phase and lipid in the gel phase. At the bottom: force-distance measurement plots on gel phase lipid

$10 \mathrm{~nm}$ (De Angelis et al., 2010a). We expect that the tip will be able to scan also biological samples with reasonable thickness.

\section{Biological Samples}

Biological samples are generally delicate, extremely soft and have a low adhesion force to the substrate. For these reasons particular care has to be taken during imaging. High resolution imaging of soft biomolecules is better achieved in wet conditions to eliminate the attractive capillary forces in the water meniscus present between the tip and the sample. Otherwise particular requirements have to be adopted to resolve nanometer features (Poma et al., 2005). In general, the lateral stress applied in contact mode operation of a scanning AFM tip can easily damage the sample whereas the lateral shear in the tip-sample interaction is significantly reduced in dynamic mode. For these reasons this method is generally preferred in AFM biological applications (Hansma et al., 1994). We therefore used a dynamic mode approach with amplitude setpoint and feedback gain set to prevent harsh interactions between tip and sample.

Lipid Bilayers. Model membranes, such as liposomes and supported lipid bilayers mimic the behavior of the lipid part of the cell membrane. They are soft
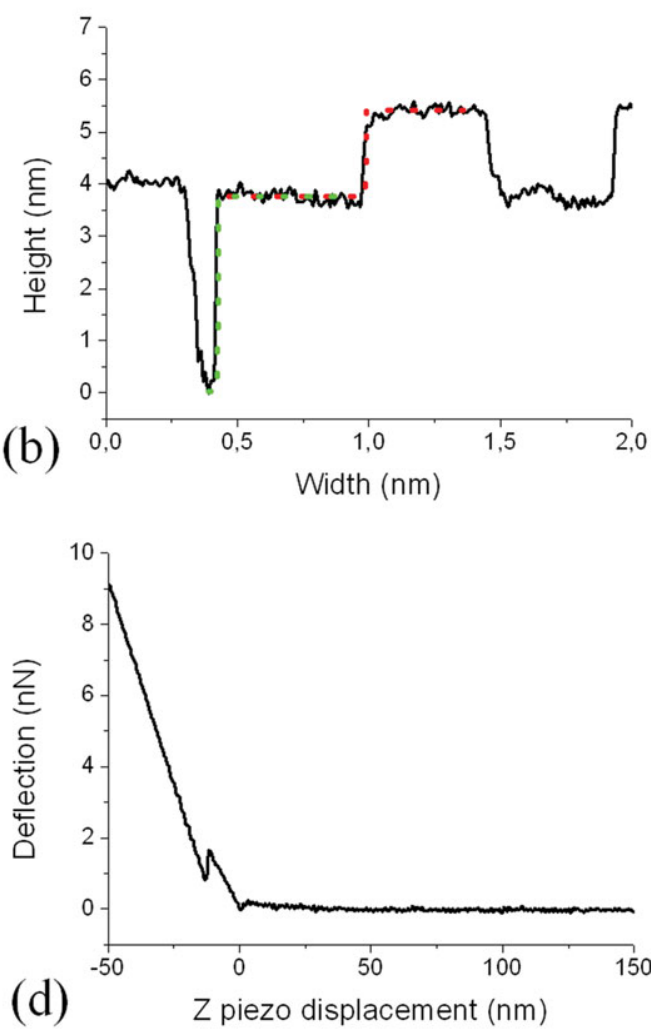

(c) indicated by arrow point no. 2 in (a) and on fluid phase lipid (d) indicated by arrow point no. 3 in (a). Inset scale bar is $1 \mu \mathrm{m}$. [Color figure can be viewed in the online issue, which is available at wileyonlinelibrary.com.]

and delicate samples as long as their structure is maintained by weak noncovalent interaction and their fluidity is preserved also in the presence of a solid substrate. In the study of these systems, AFM has been applied to explore the fine structure and physicochemical properties of physiologically relevant membranes (Canale et al., 2010; Garcia-Manyes et al., 2005; Nussio et al., 2009), to investigate the interaction between membranes and pathological proteins (Chiantia et al., 2008; Dante et al., 2011) and to study the stability or efficiency to fuse liposomes used in drugs delivery (Nakano et al., 2008). As a starting point, we evaluated the nanocone tip performance on the liquid/gel SLBs domain of a POPC/DPPC lipid mixture formed on mica. We believe this sample is first of all a good test for mimicking a soft biological material that the tip should scan without damage. Moreover, the lipid domains configured into two phases represent a good challenge test for evaluating tip lateral sensitivity. Phase separated SLB's are characterized by the coexistence of fluid and crystalline (gel) domains. The nanocone tip evidences the differences among the two domains, both in topography and force measurements ( $4 \mu \mathrm{m}$ scan size). When looking at topography image (Fig. 2a), areas of two different colors differentiate the fluid and the gel phase: the separation is sharp and there is no evidence of 


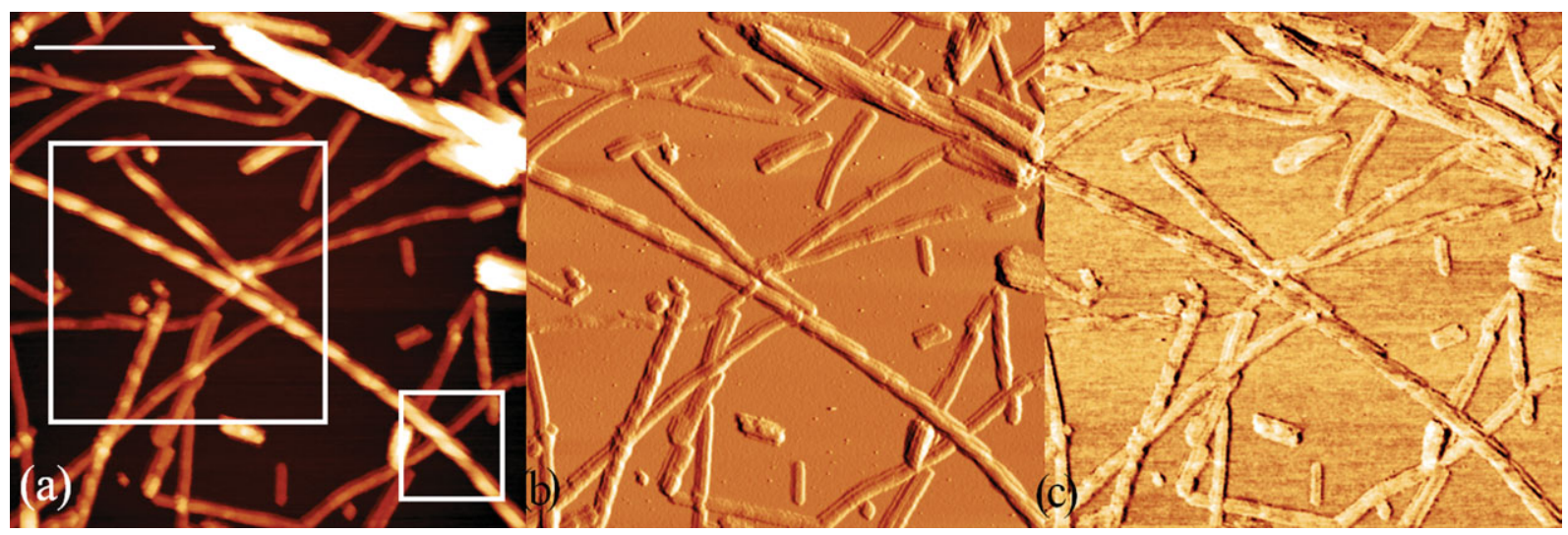

Fig. 3. (a) topography, (b) amplitude signal, and (c) phase signal of insulin sample in liquid. 843.8 $\mathrm{nm}$ scan revealing several features. Inset scale bar is $300 \mathrm{~nm}$. [Color figure can be viewed in the online issue, which is available at wileyonlinelibrary.com.]

shear or tip contamination effects. The profile of the section number 1 (plot in Fig. 2b) shows that the step height between the substrate and the POPC is $4.0 \pm$ $0.10 \mathrm{~nm}$ and the step height between the POPC and DPPC is $1.5 \pm 0.12 \mathrm{~nm}$, fairly in accordance with previous measurements (Dufrêne et al., 1997; Giocondi et al., 2001) and with crystallographic data (Kucerka et al., 2005). In force spectroscopy measurements a graph showing an extend and a retract curve describes the tip approaching and retracting from the surface. If a lipid bilayer is formed on a rigid substrate such as mica or silicon, one or more discontinuities can appear on the extend curve, where the tip is indenting the lipid layer with a force greater than the membrane can withstand (Garcia-Manyes and Sanz, 2010). Force spectroscopy curves on POPC/DPPC lipid bilayers showed a reproducible behavior. As an example two force spectroscopy points were chosen one in the gel phase (arrow point no. 2 in Fig. 2a) and the other in the fluid phase (arrow point no. 3 in Fig. 2a). The extend curves (plot in Figs. 2c and 2d) show a jump corresponding to a bilayer rupture when the tip is approaching the sample. The breakthrough force needed is different when indenting the lipid in gel phase and fluid phase. In detail, gel phase bilayer has an indentation peak between 4 and $6 \mathrm{nN}$ (Fig. 2c), while fluid phase bilayer has an indentation peak at lower applied forces, between 2 and $3 \mathrm{nN}$ (Fig. 2d). As expected the higher order in the packing of the hydrophobic lateral chain of the lipid in the gel phase gives rise to a more stable membrane. Given these clear results, we can conclude that the nanocone tip is performing well on soft model membranes: its imaging capability is not limited by tip-sample interaction and contors the phase separated lipids in reliable way.

Amyloid Fibrils. Amyloid aggregates are fibrillar assemblies that generate upon polymerization of peptides and proteins. Amyloid aggregation can be obtained in vitro, under conditions that destabilize the native state of the protein. In water solution at low $\mathrm{pH}$ and high temperature insulin is well known to form fibrils generated by the juxtaposition of a variable number of protofilaments. At longer aggregation time fibrils undergo higher order of aggregation. The accumulation of fibrillar structures in tissues and organs is the hallmark of an entire class of pathologies generally known as amyloidosis. Mature amyloid fibrils are formed from the association of small protofilaments. It is reported that the complexity of human insulin fibril aggregation can reach the level of bundle of fibrils or cluster of fibrils and next cluster of bundles (Manno et al., 2007). Therefore, measuring mature samples we expect to observe several fibrillar structures characterized by different size and shape, corresponding to different aggregation states of the protein, which can give a contribution to reveal the performance capability of the tip under study. Most of the works presented in literature reported results obtained on dried samples: in this case fibrils overcome a significant shirking due to dehydration and their structure can be affected by this procedure. Many are the AFM studies performed in liquid (Blackley et al., 2000; Fukuma et al., 2008; Goldsbury et al., 1999), but among them only a few showed high-resolution images acquired by standard tapping mode AFM (Chamberlain et al., 2000; van Raaij et al., 2006). In this article we show insulin fibrils substructure, with subnanometric resolution, obtained operating in liquid on hydrated samples. The fibrillation ability of human insulin was here exploited to produce complex nanostructures in vitro with small effort. Image in Figure 3a shows three $843.8 \mathrm{~nm}$ scan images obtained on mature fibrils deposited on mica working in fluid environment. As expected, the states of aggregation are different, showing different types of bundles, with diameters spanning from tens of nanometers to few $\mathrm{nm}$ and different structural features. Twist repeats both left- and right-handed are present in height topography (Fig. 3a), which can be better appreciated in the amplitude error signal and phase signal (Figs. $3 \mathrm{~b}$ and $3 \mathrm{c}$ ). Handedness of protein fibrillar aggregates has been reported to be usually left side oriented, even if, right-handed twists are reported for proteins bearing D-aminoacids (Harper et al., 1997). In fact, in this study we observed an almost complete occurrence of left-handed fibrils, particularly when considering smallest features, whereas, like shown in this image, higher order of aggregation can present also righthanded twist (top right structure in Fig. 3a). Together with twist structure, in the sample is also present a ribbon-like aggregation type, which is consistent with 

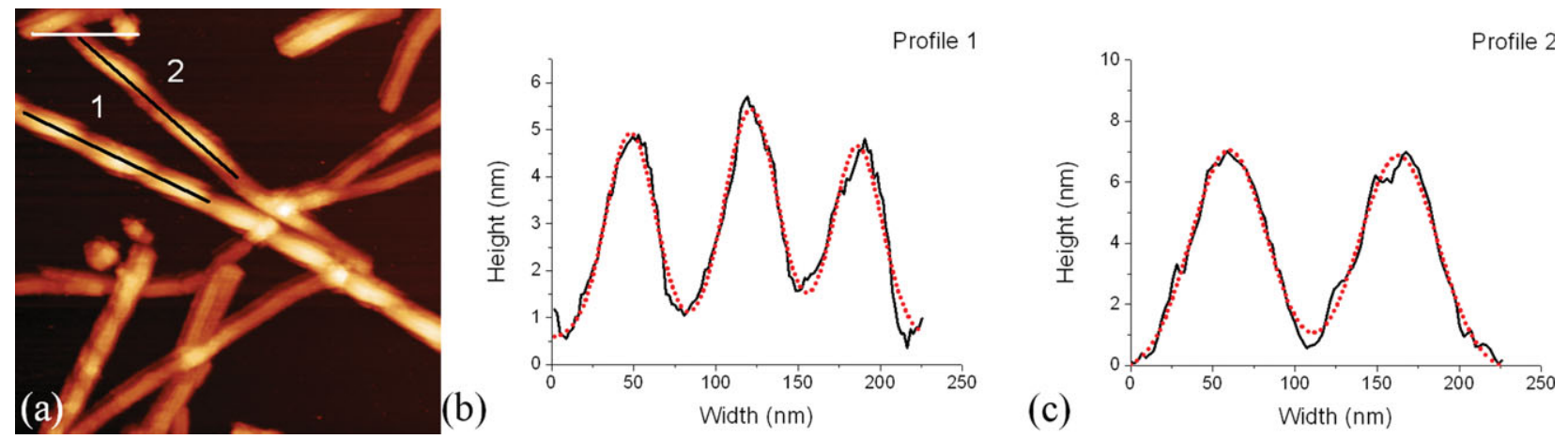

Fig. 4. (a) Topography of the $407 \mathrm{~nm}$ area corresponding to the major square of Fig. 2a. Lines 1 and 2 in (a) correspond to longitudinal cross-sections depicted in (b) and (c) as profiles and fitted to a Gaussian model to show peak-to-peak distance. Inset scale bar is $100 \mathrm{~nm}$.
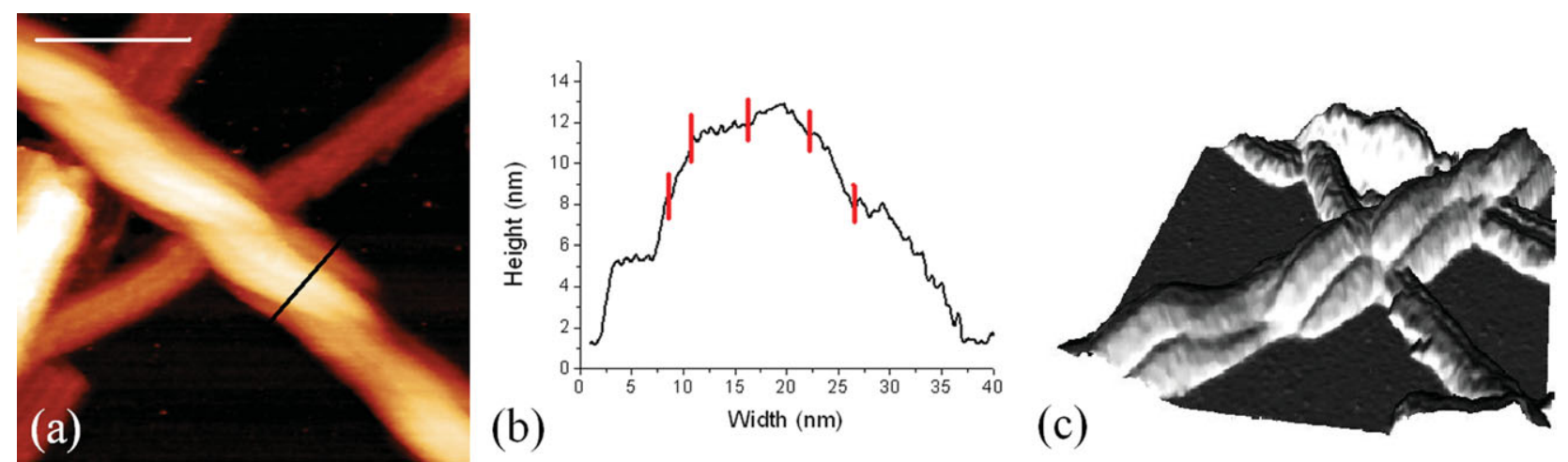

Fig. 5. (a) Topography image corresponding to the minor square area in Fig. 2a and (b) orthogonal section of the major bundle (black line). Vertical red lines show the profile discontinuities evidencing the fibrillar substructures. (c) Three-dimensional projection of topography image in (a) after a rotation of $90^{\circ}$ on main axis. Inset scale bar is $50 \mathrm{~nm}$.

previous studies that reported the tendency of several proteins to aggregate in both twist- and ribbon-like features in the same preparation.

We choose to characterize two fibrils among the many showing a difference in crossover distance. A zoomed-in topography image of $407 \mathrm{~nm}$ was acquired (big square in Fig. 3a reported in Fig. 4a) and two longitudinal sections were drawn. A periodicity of 47 and $105 \mathrm{~nm}$ for the two twisted structures (black line in profile 1 and 2 in Fig. 4) is confirmed by fitting with a Gaussian curve the height sections (dotted red line in Fig. 4 profile 1-2). In the same topography image a $70 \mathrm{~nm}$ periodic fibril structure was also present (section not shown).

The periodicity measurements are well following the data reported in literature for insulin fibrils. When scanning a zoomed area (small square at the bottom right of Fig. 2a reported in Fig. 5a), the orthogonal profile of the twist-like fibril is $35-\mathrm{nm}$ wide and $10-\mathrm{nm}$ high (Fig. 5b). The asymmetrical cross-section is consistent with the loosening attributed by Chamberlain (2000) to soft biological samples in liquid. The substructures of the amyloid fibrils are clearly displayed in Figure 5b. Features with cross-section of about $4 \mathrm{~nm}$ are revealed (vertical bars in the profile plotted in Fig. $5 b)$ : this clearly evidences the capability of the tip to provide topographical data with unprecedented lateral resolution, working in liquid. These data are well in accordance with several findings about the real cross-section of the amyloid fibril protofilament measured with various techniques, spanning from TEM, cryo EM, AFM on dried and hydrated samples which is reported to be in the range of 2-5 $\mathrm{nm}$ (Chamberlain et al., 2000). Going into specific, insulin amyloid protofilament has been reported to have cryo EM cross-section around 3$4 \mathrm{~nm}$ (Jiménez et al., 2002) and this is consistent with our AFM measurements performed on hydrated samples. Except for few works (Chamberlain et al., 2000) to date there are no reports of such nanometric resolution able to discriminate among protofilaments working in liquid environment. The protofilament structure is more evident if the topography in Figure $5 \mathrm{a}$ is plotted as a three dimensional projection: the protofilaments forming the fibrils are clearly visible particularly in the major bundle (Fig. 5c).

We used the small feature in Figure $5 \mathrm{a}$ to estimate the tip radius of curvature by assuming that the twisted fibril is a tube with a diameter equal to the height measured in AFM topography. Comparing this value to the width of the same fibril obtained in topography image we estimated a tip radius of curvature of $10 \mathrm{~nm}$. This value should be an underestimation of the tip quality considering the broadening propensity of bi- 
ological materials on the substrate when measured in liquid. Also we found a similar value $(11.8 \mathrm{~nm})$ while imaging the tip before use in scanning electron microscopy (SEM) (inset of Fig. 1b). After AFM measurements, which included force spectroscopy (the probe comes to contact with the surface), the tip was directly imaged in SEM without further preparation. The radius of curvature of the nanocone tip apex was found to be roughly around $30 \mathrm{~nm}$ (data not shown). The tip appeared free of contaminants or debris even after working with biological material in liquid. This is also consistent with our results while performing force-distance curves on the same insulin fibril sample: minor adhesion between the tip and the sample was found, whereas higher adhesion values were found using commercial silicon nitride tip (data not shown). Moreover the contribution of the tip material to its performance is also proven by the absence of electrostatic repulsion when the tip is in the approaching part of the curve (see Engel, 2011 for examples). We can speculate about the reason why the tip surface remains clean when interacting with the sample, which is also very likely related, together with the tip radius of curvature, to its ability to perform high-resolution topography. The nanocone tip is made of a mixture of Platinum and Carbon that directly derive from the gas injected $\left((\mathrm{CH} 3)_{3} \mathrm{Pt}\left(\mathrm{C}_{\mathrm{p}} \mathrm{CH}_{3}\right)\right)$. The metal is deposited as clusters of small $\mathrm{nm}$ sized particles embedded in amorphous carbon (graphite) (De Angelis et al., 2011b). The nanostructured surface and the composition are contributing to the tip surface microscopic properties, possibly making the tip surface less prone to interact with hydrated samples.

Titin Force Spectroscopy. Finally we wanted to verify that the cantilever/nanocone is performing well also in a classical experiment of polydomain proteins unfolding. In this experiment the nanocone tip was coated with $10 \mathrm{~nm}$ gold layer to favor its adhesion to the protein (gold interacts with thiol groups of the protein cysteine residues). I27O is a recombinant polyprotein made of eight Ig repeats that have a precise force spectroscopy signature. Hence, the force-extension relationship shows a characteristic curve with a sawtooth profile with as many peaks as the number of domains (Rief, 1997). In an ideal experiment eight domains should be unfolded. In the nanocone experiment the majority of the pullings was of two to seven domains and some experiments showed an increase of the number of domains pulled versus a decrease in the contor length calculated. This was possibly due to the pulling of two or more proteins at the same fishing point. In the following plot (Fig. 6) the unfolding path of a single $\mathrm{I} 27 \mathrm{O}$ protein pulled with a gold coated nanocone tip is shown. The force extension segments can be explained as stepwise increases in the contor length of a polymer whose elastic properties can be described by a worm-like chain model. In detail, at pulling velocity of $0.5 \mu \mathrm{m} \mathrm{s}^{-1}$, a force of about $450 \mathrm{pN}$ is elongating each domain of $28 \mathrm{~nm}$ giving a peak-to-peak distance of $25 \mathrm{~nm}$. This result is consistent with the data reported by Carrion-Vazquez in his comparison of mechanical and chemical unfolding of the I27O AFM reference protein (Carrion-Vazquez, 1999). The fabrication procedure did not altered the cantilever stability and the gold coated tip provided a good pulling efficiency, in particular on proteins with reactive cystein groups.

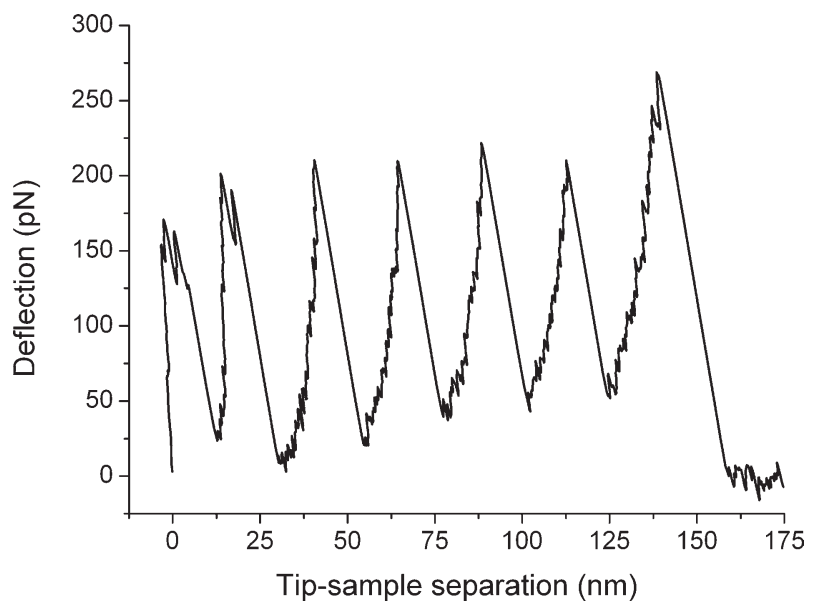

Fig. 6. Force spectroscopy pulling of a I27O recombinant polyprotein. While the tip is retracting from the sample the polydomain protein is responding with an opposite force to the pulling. The unfolding profile is depicted as a force/distance curve.

\section{CONCLUSIONS}

The chemical fingerprint of biomolecules in their natural environment down to nanometer resolution is a task of several research groups. Many efforts are being made towards the coupling of Raman and AFM spectroscopy techniques to obtain data on single biomolecules such as e.g., RNA lipid bilayers and insulin fibrils (Bailo and Deckert, 2008; Opilik et al., 2011; Moretti et al. 2012). However TERS probes that demonstrate electromagnetic field enhancement often lack the ability to topographically resolve nanometer features, particularly when the analysis is performed in liquid phase. Several AFM probes have been developed for high resolution imaging (including nanotubes), however, to the best of our knowledge, usually high performance tips are either quite fragile and/or debris accumulate easily on their surface, being this a drawback for parallel collection of Raman signal. On the contrary, our nanofabrication approach provides a stable nanostructure less prone to rupture or to debris accumulation. This property allowed us to collect several high quality topography images before the tip looses its resolution without perturbing the molecule aggregation state. This result is particularly significant when working on biological samples that have a limited shelf life, taking into consideration that long time or multipoint acquisition data are often necessary to have a complete Raman spectra map. Regarding the topography resolution capability, high performance was revealed investigating both lipid bilayers and insulin fibrils in liquid phase. In fact, to date, there are few high quality topography reports on amyloid fibrils in liquid and none of them shows such a clear pattern $(<1$ $\mathrm{nm}$ resolution). The tip apex radius of curvature is fundamental for obtaining high-resolution topography, however we guess that also the surface chemical properties of the tip play a role in its ability to finely scan the sample. When gold coated, the tip was also able to efficiently perform force-distance curves on polydomain proteins. We foresee future performances on more complex systems such as native membrane proteins, proteins embedded in lipid bilayers up to membrane 
extracted from cells and cells themselves. This will also include experiments of force-distance curves on the same systems. The claimed high resolution capability of the tip is required when it is coupled to a Raman spectroscopy device in SERS and TERS-like setup (De Angelis et al., 2010a, 2011a). Thus, in addition to the previously established field enhancement effect of the nanofabricated probe (Bek et al., 2010; De Angelis et al., 2010a,b), here we clearly demonstrated the stability of the nanocone and its excellent quality topography on fine biomolecules. These promising results put us on the way of the single molecule AFM and Raman characterization of biological samples in liquid.

\section{REFERENCES}

Allen S, Chen X, Davies J, Davies MC, Dawkes AC, Edwards JC, Roberts CJ, Sefton J, Tendler SJ, Williams PM. 1997. Detection of antigen-antibody binding events with the atomic force microscope. Biochemistry 36:7457-7463.

De Angelis F, Das G, Candeloro P, Patrini M, Galli M, Bek A, Lazzarino M, Maksymov I, Liberale C, Andreani LC, Di Fabrizio E. 2010a. Nanoscale chemical mapping using three-dimensional adiabatic compression of surface plasmon polaritons. Nat Nanotechnol 5:67-72.

De Angelis F, Gentile F, Mecarini F, Das G, Moretti M, Candeloro P, Coluccio ML, Cojoc G, Accardo A, Liberale C, Proietti Zaccaria R, Perozziello G, Tirinato L, Toma A, Cuda G, Cingolani R, Di Fabrizio E. 2011a. Breaking the diffusion limit with superhydrophobic delivery of molecules to plasmonic nanofocusing SERS structures. Nat Photon 5:682-687.

De Angelis F, Liberale C, Coluccio ML, Cojoc G, Di Fabrizio E. $2011 \mathrm{~b}$. Emerging fabrication techniques for $3 \mathrm{D}$ nano-structuring in plasmonics and single molecule studies. Nanoscale 3:2689-2696.

De Angelis F, Patrini M, Das G, Maksymov I, Galli M, Businaro L, Andreani LC, Di Fabrizio E. 2008. A hybrid plasmonic-photonic nanodevice for label-free detection of a few molecules. Nano Lett 8:2321-2327.

De Angelis F, Pujia A, Falcone C, Iaccino E, Palmieri C, Liberale C, Mecarini F, Candeloro P, Luberto L, de Laurentiis A, Das G, Scala G, Di Fabrizio E. 2010b. Water soluble nanoporous nanoparticle for in vivo targeted drug delivery and controlled release in $\mathrm{B}$ cells tumor context. Nanoscale 2:2230-2236.

Bailo E, Deckert V. 2008. Tip-enhanced Raman spectroscopy of single RNA strands: Towards a novel direct-sequencing method. Ang Chem Int Ed 47:1658-1661.

Bek A, De Angelis F, Das G, Di Fabrizio E, Lazzarino M. 2010. Tip enhanced Raman scattering with adiabatic plasmon focusing tips. Micron 42:313-317.

Blackley HK, Sanders GH, Davies MC, Roberts CJ, Tendler SJ, Wilkinson MJ. 2000. In situ atomic force microscopy study of betaamyloid fibrillization. J Mol Biol 298:833-840.

Canale C, Jacono M, Diaspro A, Dante S. 2010. Force spectroscopy as a tool to investigate the properties of supported lipid membranes. Micros Res Tech 73:965-972.

Canale C, Torre B, Ricci D, Braga PC. 2011. Recognizing and avoiding artifacts in atomic force microscopy imaging. Method Mol Biol 736:31-43.

Carrion-Vazquez M. 1999. Mechanical and chemical unfolding of a single protein: A comparison. Proc Natl Acad Sci USA 96:3694-3699.

Chamberlain AK, MacPhee CE, Zurdo J, Morozova-Roche LA, Hill HA, Dobson CM, Davis JJ. 2000. Ultrastructural organization of amyloid fibrils by atomic force microscopy. Biophys $\mathrm{J}$ 79:3282-3293.

Chiantia S, Ries J, Chwastek G, Carrer D, Li Z, Bittman R, Schwille P. 2008. Role of ceramide in membrane protein organization investigated by combined AFM and FCS. Biochim Biophys Acta 1778:1356-1364.

Chtcheglova LA, Waschke J, Wildling L, Drenckhahn D, Hinterdorfer P. 2007. Nano-scale dynamic recognition imaging on vascular endothelial cells. Biophys J 93:L11-L13.

Dante S, Hauß T, Steitz R, Canale C, Dencher NA. 2011. Nanoscale structural and mechanical effects of beta-amyloid (1-42) on polymer cushioned membranes: A combined study by neutron reflectometry and AFM force spectroscopy. Biochim Biophys Acta 1808:2655-2646.

Das G, Mecarini F, Gentile F, De Angelis F, Mohan Kumar H, Candeloro P, Liberale C, Cuda G, Di Fabrizio E. 2009. Nano-pat- terned SERS substrate: Application for protein analysis vs. temperature. Biosens Bioelectron 24:1693-1699.

DeRose J. 1998. Studying the surface of soft materials (live cells) at high resolution by scanning probe microscopy: Challenges faced. Thin Solid Films 331:194-202.

Descrovi E, Aeschimann L, Soboleva I, De Angelis F, Giorgis F, Di Fabrizio E. 2009. High resolution capabilities of all-silica cantilevered probes for near-field optical microscopy. J Nanosci Nanotechnol 9:6460-6464.

Dufrêne YF, Barger WR, Green J-BD, Lee GU. 1997. Nanometerscale surface properties of mixed phospholipid monolayers and bilayers. Langmuir 13:4779-4784.

Dufrêne YF, Evans E, Engel A, Helenius J, Gaub HE, Müller DJ. 2011. Five challenges to bringing single-molecule force spectroscopy into living cells. Nat Methods 8:123-127.

Engel A. 2011. Imaging and interrogating native membrane proteins using the atomic force microscope. Methods Mol Biol 736: 153-167.

Fleischer M, Zhang D, Braun K, Jäger S, Ehlich R, Häffner M, Stanciu C, Hörber JKH, Meixner AJ, Kern DP. 2010. Tailoring gold nanostructures for near-field optical applications. Nanotechnology 21:065301.

Fukuma T, Mostaert AS, Serpell LC, Jarvis SP. 2008. Revealing molecular-level surface structure of amyloid fibrils in liquid by means of frequency modulation atomic force microscopy. Nanotechnology 19:384010.

Garcia-Manyes S, Sanz F. 2010. Nanomechanics of lipid bilayers by force spectroscopy with AFM: A perspective. Biochim Biophys Acta 1798:741-749.

Garcia-Manyes S, Oncins G, Sanz F. 2005. Effect of ion-binding and chemical phospholipid structure on the nanomechanics of lipid bilayers studied by force spectroscopy. Biophys J 89:18121826.

Giocondi M-C, Vié V, Lesniewska E, Milhiet P-E, Zinke-Allmang M, Le Grimellec C. 2001. Phase topology and growth of single domains in lipid bilayers. Langmuir 17:1653-1659.

Goldsbury C, Kistler J, Aebi U, Arvinte T, Cooper GJ. 1999. Watching amyloid fibrils grow by time-lapse atomic force microscopy. J Mol Biol 285:33-39.

Hansma PK, Cleveland JP, Radmacher M, Walters DA, Hillner PE, Bezanilla M, Fritz M, Vie D, Hansma HG, Prater CB, Massie J, Fukunaga L, Gurley J, Elings V. 1994. Tapping mode atomic force microscopy in liquids. Appl Phys Lett 64:1738.

Harper JD, Lieber CM, Lansbury PT Jr. 1997. Atomic force microscopic imaging of seeded fibril formation and fibril branching by the Alzheimer's disease amyloid- $\beta$ protein. Chem Biol 4:951-959.

Hinterdorfer P. 1996. Detection and localization of individual antibody-antigen recognition events by atomic force microscopy. Proc Natl Acad Sci USA 93:3477-3481.

Hutter JL, Bechhoefer J. 1993. Calibration of atomic-force microscope tips. Rev Sci Instrum 64:1868.

Jiménez JL, Nettleton EJ, Bouchard M, Robinson CV, Dobson CM, Saibil HR. 2002. The protofilament structure of insulin amyloid fibrils. Proc Natl Acad Sci USA 99:9196-9201.

Kharintsev SS, Noskov AI, Hoffmann GG, Loos J. 2011. Nearfield optical taper antennas fabricated with a highly replicable ac electrochemical etching method. Nanotechnology 22:025202.

Kienberger F, Kada G, Mueller H, Hinterdorfer P. 2005. Single molecule studies of antibody-antigen interaction strength versus intra-molecular antigen stability. J Mol Biol 347:597-606.

Kucerka N, Tristram-Nagle S, Nagle JF. 2005. Structure of fully hydrated fluid phase lipid bilayers with monounsaturated chains. J Membr Biol 208:193-202.

Liberale C, Minzioni P, Bragheri F, De Angelis F, Di Fabrizio E, Cristiani I. 2007. Miniaturized all-fiber probe for three-dimensional optical trapping and manipulation. Nat Photon 1:723-727.

Manno M, Craparo EF, Podestà A, Bulone D, Carrotta R, Martorana V, Tiana G, San Biagio PL. 2007. Kinetics of different processes in human insulin amyloid formation. J Mol Biol 366:258 274.

Mingeot-Leclercq M-P, Deleu M, Brasseur R, Dufrêne YF. 2008. Atomic force microscopy of supported lipid bilayers. Nat Protocols 3:1654-1659.

Moretti M, Di Fabrizio E, Cabrini S, Musetti R, De Angelis F, Firrao G. 2008. An ON/OFF biosensor based on blockade of ionic current passing through a solid-state nanopore. Biosens Bioelectron 24:141-147.

Moretti M, Proietti Zaccaria R, Descrovi E, Das G, Leoncini M, Liberale C, De Angelis F, Di Fabrizio E. 2012. Reflection-mode TERS on insulin amyloid fibrils with top-visual AFM probes. Plasmonics Epub ahead of print: DOI: 10.1007/s11468-012-9385-x. 
Nakano K, Tozuka Y, Yamamoto H, Kawashima Y, Takeuchi H. 2008. A novel method for measuring rigidity of submicron-size liposomes with atomic force microscopy. Int J Pharm 355:203-209.

Nussio MR, Oncins G, Ridelis I, Szili E, Shapter JG, Sanz F, Voelcker NH. 2009. Nanomechanical characterization of phospholipid bilayer islands on flat and porous substrates: A force spectroscopy study. J Phys Chem B 113:10339-10347.

Oberhauser AF, Marszalek PE, Erickson HP, Fernandez JM. 1998. The molecular elasticity of the extracellular matrix protein tenascin. Nature 393:181-185.

Opilik L, Bauer T, Schmid T, Stadler J, Zenobi R. 2011. Nanoscale chemical imaging of segregated lipid domains using tip-enhanced Raman spectroscopy. Phys Chem Chem Phys 13:9978-9981.

Poma A, Spanò L, Pittaluga E, Tucci A, Palladino L, Limongi T. 2005. Interactions between saporin, a ribosome-inactivating protein, and DNA: A study by atomic force microscopy. J Microsc 217:69-74.

Puchner EM, Gaub HE. 2009. Force and function: Probing proteins with AFM-based force spectroscopy. Curr Opin Struct Biol 19:605614.

Pérennès F, Marmiroli B, Matteucci M, Tormen M, Vaccari L, Di Fabrizio E. 2006. Sharp beveled tip hollow microneedle arrays fabricated by LIGA and 3D soft lithography with polyvinyl alcohol. J Micromech Microeng 16:473-479.

Rief M. 1997. Reversible unfolding of individual titin immunoglobulin domains by AFM. Science 276:1109-1112.

Stadler J, Schmid T, Zenobi R. 2010. Nanoscale chemical imaging using top-illumination tip-enhanced Raman spectroscopy. Nano Lett 10:4514-4520.
Stroh CM, Ebner A, Geretschläger M, Freudenthaler G, Kienberger F Kamruzzahan ASM, Smith-Gill S.J Gruber H.J Hinterdorfer P. 2004. Simultaneous topography and recognition imaging using force microscopy. Biophys J 87:1981-1990.

Tormen M, Businaro L, Altissimo M, Romanato F, Cabrini S, Pérennès F, Proietti R, Sun H-B, Kawata S, Di Fabrizio E. 2004. 3D patterning by means of nanoimprinting, X-ray and twophoton lithography. Microelectron Eng 73/74:535-541.

van Raaij ME, Segers-Nolten IMJ, Subramaniam V. 2006. Quantitative morphological analysis reveals ultrastructural diversity of amyloid fibrils from alpha-synuclein mutants. Biophys J 91:L96L98.

Velegol SB, Pardi S, Li X, Velegol D, Logan BE. 2003. AFM imaging artifacts due to bacterial cell height and AFM tip geometry. Langmuir 19:851-857.

Weber-Bargioni A, Schwartzberg A, Cornaglia M, Ismach A, Urban JJ, Pang Y, Gordon R, Bokor J, Salmeron MB, Ogletree DF, Ashby P, Cabrini S, Schuck PJ. 2011. Hyperspectral nanoscale imaging on dielectric substrates with coaxial optical antenna scan probes. Nano Lett 11:1201-1207.

Wong J. 1999. Direct force measurements of the streptavidin-biotin interaction. Biomol Eng 16:45-55.

Yacoot A, Koenders L. 2008. Aspects of scanning force microscope probes and their effects on dimensional measurement. J Phys D Appl Phys 41:103001.

Yu AA, Savas T, Cabrini S, Difabrizio E, Smith HI, Stellacci F. 2005. High resolution printing of DNA feature on poly(methyl methacrylate) substrates using supramolecular nano-stamping. J Am Chem Soc 127:16774-16775. 